\title{
Nonlinear Finite Element Modeling of Reinforced Concrete Structures under Impact Loads
}

\author{
by Selcuk Saatci and Frank J. Vecchio
}

The methods available in the literature for the analysis of reinforced concrete $(R C)$ structures subjected to impact loads generally exhibit some deficiencies in aspects relating to applicability, practicality, and accuracy. The shear-dominant behavior of RC members under impact loads creates another significant shortcoming, because modeling the shear behavior of RC has long been a challenging issue. This study aims to present and verify a nonlinear finite element analysis procedure employing the Disturbed Stress Field Model, based on a smeared rotating crack approach, as an advanced method of modeling shear behavior under impact conditions. The proposed methodology has a wide range of applicability, and displays fast solution time while providing extensive and accurate information on structural behavior. The methodology was tested by analyzing a set of RC beams subjected to impact loads. A high level of accuracy was demonstrated in various comparisons between test and analysis results, including peak and residual displacements, crack profiles, and reinforcement strains.

Keywords: impact behavior; impact loading; nonlinear finite element analysis; shear.

\section{INTRODUCTION}

The demand for impact resistant design of reinforced concrete (RC) structures crosses a wide spectrum, from nuclear power reactors to protective barriers and industrial facilities to rock sheds. Moreover, as a result of recently elevated terror threat levels in the world, the impact resistant design of buildings has become a new focus of attention. As a result, the analysis and design of RC structures for impact loads has been an objective of many researchers and designers.

Although a wide range of methods are used in current practice, it is difficult to name a single commonly accepted method for the design of RC structures against impact loads. Some existing methods offer relatively practical formulations and recommendations; however, they tend to put severe limitations on the type and geometry of the structure modeled in order to simplify the formulation of the impact problem. One such well-known method, for example, reduces the analyzed structure to a single-degree-of freedom (SDOF) system, modeling the structure as a mass attached to a spring, representing its stiffness. ${ }^{1}$ Although this approach has been followed and refined by several researchers, ${ }^{2,3}$ its limited applicability only allows the impact analysis of simple structures, such as isolated beams and slabs that can be reduced to an SDOF system. Even for such structures, only the displacementtime response of a representative point on the analyzed structure can be obtained, with no information on the stress distribution along the member. To the contrary, because of the high dynamic conditions present in cases of an impact loading, the geometry and inertia of the structure play important roles. Ignoring such effects for the sake of simplifying the problem typically leads to inaccurate or misleading information about the impact behavior of the structure analyzed. Unsafe or inefficient designs are the eventual outcome.
To overcome the limitations of simplistic methods, designers usually resort to more advanced numerical modeling methods, such as the finite element method, to address the complexities of the design problem at hand and thus obtain more accurate solutions. The majority of the finite element packages commonly used in practice for the impact analysis of RC structures, however, require undertaking sophisticated solutions of a contact problem between two colliding objects modeled with high detail. ${ }^{4,5}$ Although such an approach removes the limitations on the geometry of the structure and allows the analysis of a wide range of impact load cases, it also creates major disadvantages for practical design purposes when the complexity of the modeling and the length of computation time are considered. Moreover, modeling the behavior of RC has always proven to be challenging in many aspects. In particular, the shear behavior of concrete is a complex issue, and the lack of rational methods for representing such behavior has long been a major deficiency in the analytical modeling of RC. On the other hand, shear mechanisms are known to dominate the overall behavior of concrete structures subjected to impact loads. ${ }^{6,7}$ Hence, an accurate modeling of the shear behavior is crucial in predicting the impact response of such structures.

This paper introduces and verifies a nonlinear finite element analysis (NLFEA) procedure, which eliminates the limitations of simplistic methods while avoiding sophisticated and time consuming techniques found in more esoteric finite element approaches. The proposed procedure employs stateof-the-art techniques for modeling the shear behavior of reinforced concrete, and provides for a widely applicable tool with good accuracy and fast solution time.

To demonstrate the application of the procedure, an RC static NLFEA program called VecTor $2^{8}$ was modified to incorporate dynamic analysis capabilities for impact loads. VecTor2 uses a smeared rotating crack approach based on the disturbed stress field model ${ }^{9}$ (DSFM) for its computational methodology; the DSFM is an extension of the modified compression field theory ${ }^{10}$ (MCFT) reconfigured to consider crack slip and noncoinciding stress and strain principal axes. The MCFT and DSFM have been developed as rational methods to model the shear behavior of RC structures and, over the years, have been successfully applied in accurately simulating the behavior of numerous RC structures under static loads. The current study applies DSFM to the case of impact loads, for which the shear behavior was shown to play a major role.

ACI Structural Journal, V. 106, No. 5, September-October 2009

MS No. S-2008-329 received October 7, 2008, and reviewed under Institute publication policies. Copyright (C) 2009, American Concrete Institute. All rights reserved, including the making of copies unless permission is obtained from the copyright proprietors. Pertinent discussion including author's closure, if any, will be published in the JulyAugust 2010 ACI Structural Journal if the discussion is received by March 1, 2010. 
$\overline{\text { ACI member Selcuk Saatci is an Assistant Professor at the Izmir Institute of }}$ Technology, Izmir, Turkey. He received his BS and MS from the Middle East Technical University, Ankara, Turkey, in 1999 and 2001, respectively, and his PhD from the University of Toronto, Toronto, ON, Canada, in 2007. His research interests include the nonlinear finite element analysis and impact behavior of reinforced concrete structures.

Frank J. Vecchio, FACI, is a Professor of civil engineering at the University of Toronto. He is a member of Joint ACI-ASCE Committees 441, Reinforced Concrete Columns, and 447, Finite Element Analysis of Concrete Structures. He received the 1998 ACI Structural Research Award and the 1999 ACI Structural Engineering Award. His research interests include advanced constitutive modeling and analysis of reinforced concrete, assessment and rehabilitation of structures, and response under extreme load conditions.

In this paper, the proposed NLFEA procedure is described and the application of the technique into VecTor2 is briefly introduced. To demonstrate the effectiveness of the procedure, the NLFEA-computed responses obtained using VecTor2 are compared to the measured responses obtained from a comprehensive experimental program involving impact tests on RC beams. The comparisons include time-histories of the midspan displacements of the beams, crack profiles, and longitudinal reinforcement strains. The general applicability of VecTor2 and its computational methodology to the case of impact loads, and NLFEA-based procedures in general, are also discussed.

\section{RESEARCH SIGNIFICANCE}

Shear mechanisms usually dominate the behavior of RC members subjected to impacts. The methods currently available for the impact analysis of RC structures, however, typically fail to provide an accurate modeling of shear-related mechanisms due to their complex nature. This study shows that nonlinear finite element analysis procedures, using appropriate constitutive models for concrete such as those contained in the DSFM, ${ }^{9}$ are a viable method for advanced modeling of shear-dominated responses of RC structures under impact load conditions.

\section{NUMERICAL EVALUATION OF DYNAMIC RESPONSE}

Dynamic analysis of a structure requires establishing the equation of motion, which can be expressed as follows for a multi-degree-of-freedom structure to be solved using finite element methods

$$
m \ddot{u}+c \dot{u}+k u=p(t)
$$

where $m$ is the mass matrix, $c$ is the damping matrix, $k$ is the stiffness matrix, $p$ is the time dependent force vector, $u$ is the displacement vector, $\dot{u}$ is the velocity vector, and $\ddot{u}$ is the acceleration vector. Because Eq. (1) is derived from basic principles of mechanics, most dynamic finite element analysis methods involve its solution for $u$ in one way or another. This requires establishing the structural property matrices $m, c$, and $k$.

The mass matrix $m$ is a discrete representation of the mass of the system mobilized during the dynamic motion. In this study, for its computational ease and minimal storage requirements, a lumped mass matrix is preferred; all offdiagonal terms are zero, and the nonzero diagonal elements are simply calculated by the concentration (lumping) of the total mass of a particular portion of the structure to a single point. The concentrated mass is assigned to the associated degree of freedom, entered as the corresponding diagonal term in the mass matrix.
Determining the damping matrix $c$ from the physical state of the structure, such as the dimensions, member sizes, or materials used, is impractical, and the number of studies in this area in the literature is quite limited. Therefore, damping is usually included in the analyses not as a calculated, welldetermined physical property such as the material stiffness, but, rather, from a mathematical perspective for its ability to stabilize numerical solutions and take into account energy dissipating mechanisms. A well-known method commonly used to form a damping matrix that is both compatible with the expected damping ratios of the vibrational modes and also has practical mathematical properties is Rayleigh damping. ${ }^{11}$ The Rayleigh damping matrix is expressed as

$$
c=a_{o} m+a_{1} k
$$

where $a_{0}$ and $a_{1}$ are proportionality constants, calculated as follows

$$
a_{1}=\frac{2 \xi_{j} \omega_{j}-2 \xi_{i} \omega_{i}}{\omega_{j}^{2}-\omega_{i}^{2}}, \quad a_{0}=2 \xi_{i} \omega_{i}-a_{1} \omega_{i}^{2}
$$

where $\xi$ is the damping ratio and $\omega$ is the natural circular frequency of the two selected modes $i$ and $j$. The majority of the energy dissipating mechanisms in an RC structure, such as material hysteresis, concrete cracking and bond slippage, can be included in an NLFEA analysis through the appropriate choice of constitutive and behavioral models; for numerical considerations, however, viscous damping may also be needed in the system. Despite the fact that the proposed NLFEA procedure does not utilize modal analysis, Rayleigh damping was chosen to introduce viscous damping in this study to ensure the stability of solutions. For the calculation of the coefficients $a_{0}$ and $a_{1}$, the vibrational modes of the undamaged structure are determined using an open-code eigen-value solver, ${ }^{12}$ and damping ratios are assigned to the first and second modes to calculate $c$. A method to determine the appropriate damping ratios is discussed in the following sections.

Determination of the stiffness matrix $k$ depends on the NLFEA analysis method employed. In the present study, because of its success in modeling shear behavior, the DSFM was the selected method for representing RC behavior, the details of which can be found elsewhere. ${ }^{9}$ It should be noted that a majority of the behavioral models, such as concrete and steel hysteretic response, are incorporated into the procedures used for calculating the stiffness matrix.

Evaluating the structural response under impact loading, or any dynamic loading for that matter, usually requires the numerical integration of Eq. (1) because a closed form solution is impractical for most dynamic loads. Newmark's Method of Direct Integration ${ }^{13}$ was chosen in this study for performing such calculations. The original formulations of Newmark's Method using tangential stiffness properties, however, had to be revised as follows, because the DSFM employs a total load approach in generating a secant stiffness matrix.

The equation of motion, as in Eq. (1), can be expressed in terms of total loads for time steps $i$ and $i+1$ as follows

$$
m \ddot{u}_{i}+c \dot{u}_{i}+k_{i} u_{i}=p_{o}+p_{i}
$$




$$
m \ddot{u}_{i+1}+c \dot{u}_{i+1}+k_{i+1} u_{i+1}=p_{0}+p_{i+1}
$$

where $k$ is the secant stiffness, and $p_{0}$ is the constant force, such as self-weight. Equation (4) can be rearranged to give

$$
m\left(\ddot{u}_{i}+\Delta \ddot{u}\right)+c\left(\dot{u}_{i}+\Delta \dot{u}\right)+k_{i+1} u_{i+1}=p_{0}+p_{i+1}
$$

where $\Delta u$ is the change in displacement from the time step $i$ to $i+1$. Newmark formulations relate the incremental change in accelerations, velocities, and displacements as follow

$$
\Delta \dot{u}=\Delta t \ddot{u}_{i}+\gamma \Delta t \Delta \ddot{u}, \quad \Delta u=\Delta t \dot{u}_{i}+\frac{\Delta t^{2}}{2} \ddot{u}_{i}+\beta \Delta t^{2} \Delta \ddot{u}
$$

where $\Delta t$ is the time step length, and $\beta$ and $\gamma$ are the integration constants. Equation (6) can be substituted into Eq. (5) to obtain

$$
m\left(\ddot{u}_{i}+\Delta \ddot{u}\right)+c\left(\dot{u}_{i}+\Delta t \ddot{u}_{i}+\gamma \Delta t \Delta \ddot{u}\right)+k_{i+1} u_{i+1}=p_{0}+p_{i+1}
$$

which can be rearranged as

$$
[m+c \gamma \Delta t] \Delta \ddot{u}+m \ddot{u}_{i}+c \dot{u}_{i}+c \Delta t \ddot{u}_{i}+k_{i+1} u_{i+1}=p_{0}+p_{i+1}
$$

The incremental acceleration change in Eq. (6) can be calculated as

$$
\Delta \ddot{u}=\frac{1}{\beta \Delta t^{2}} \Delta u-\frac{1}{\beta \Delta t} \dot{u}_{i}-\frac{1}{2 \beta} \ddot{u}_{i}
$$

and Eq. (2) and (9) can be substituted in Eq. (8) and rearranged to obtain the general system of equations

$$
\begin{gathered}
{\left[k_{i+1}+\frac{1+\gamma \Delta t a_{0}}{\beta \Delta t^{2}} m+\frac{\gamma \Delta t a_{1}}{\beta \Delta t^{2}} k_{0}\right] u_{i+1}=p_{0}+p_{i+1}+} \\
{\left[\frac{1+\gamma \Delta t a_{0}}{\beta} m+\frac{\gamma \Delta t a_{1}}{\beta} k_{0}\right]\left\{\frac{u_{i}}{\Delta t^{2}}+\frac{\dot{u}_{i}}{\Delta t}+\frac{\ddot{u}_{i}}{2}\right\}-} \\
{\left[a_{0} m+a_{1} k_{0}\right]\left\{\dot{u}_{i}+\Delta t \ddot{u}_{i}\right\}-m \ddot{u}_{i}}
\end{gathered}
$$

where $k_{0}$ is the initial stiffness matrix, which is left unchanged during the entire analysis. Note that in this equation, the only unknowns at time step $i$ are $k_{i+1}$ and $u_{i+1}$. In most NLFEA procedures, $k_{i+1}$ is dependent on $u_{i+1}$; therefore, they can be solved through an iterative procedure as summarized in Fig. 1.

\section{EXPERIMENTAL PROGRAM}

To verify the proposed NLFEA procedure, an experimental program conducted at the University of Toronto ${ }^{7}$ was considered for finite element modeling. The experimental program involved eight simply supported RC beams (four pairs) tested under free-falling drop-weights impacting the specimens at the midspan. All specimens had identical longitudinal reinforcement, but with varying shear reinforcement ratios intended to investigate the effects of shear capacity on the impact behavior. Details of the test specimens are presented in Fig. 2. The specimens were impacted with two different drop-weights, a heavy weight of $600 \mathrm{~kg}(1323 \mathrm{lb})$ and a lighter weight of $211 \mathrm{~kg}$ (465 lb), to induce different levels of damage with each impact. The weights were dropped from a clear height of $3.26 \mathrm{~m}$ (128.3 in.) above the specimen, resulting in an $8.0 \mathrm{~m} / \mathrm{s}(26.25 \mathrm{ft} / \mathrm{s})$ calculated impact velocity. All specimens, with the exception of SSOb, were subjected to multiple impacts. With the a-series specimens, the beams were tested once with the lighter drop-weight and followed by two tests with the heavy one. For b-series specimens, the order was reversed; they were tested twice with the heavy drop-weight, and then one last time with the lighter drop-weight. A total of 20 impact tests were conducted. Static tests on replicas of the impact specimens were also conducted to determine their static load responses. In these tests, SS3 and SS2 demonstrated a flexural-critical behavior with a ductile response, while SS1 and SS0 were shear-critical with a brittle failure mode under the monotonically increasing static loads. A detailed description of the test program and the results can be found elsewhere. ${ }^{14}$

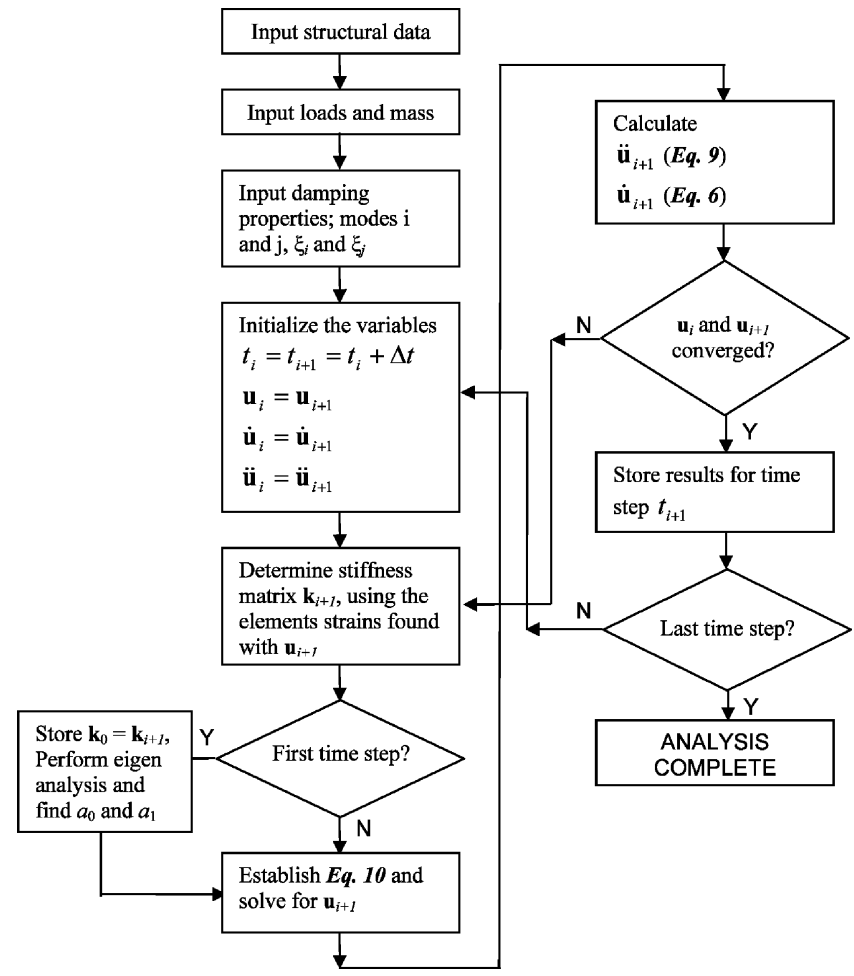

Fig. 1-Flowchart for proposed NLFEA procedure.

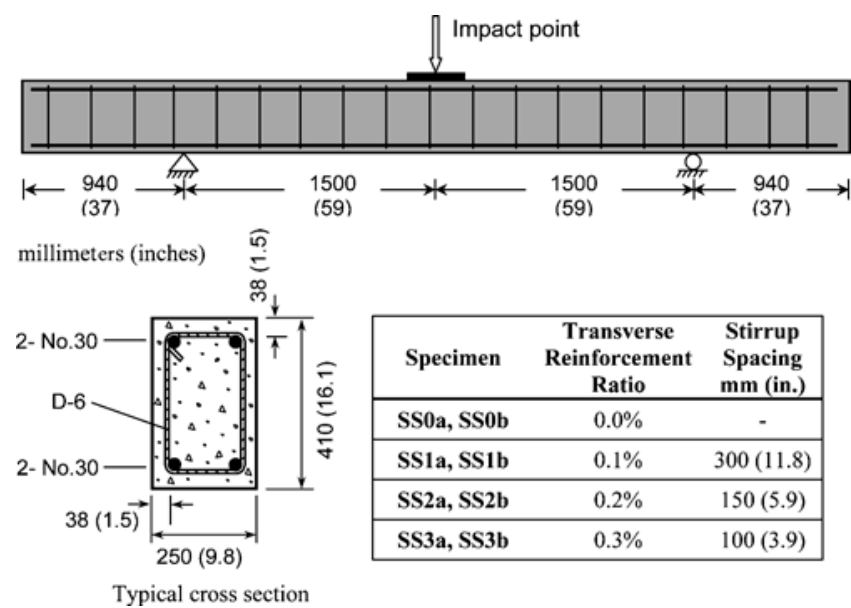

Fig. 2-Specimen properties. 


\section{FINITE ELEMENT MODELING}

For this study, the proposed NLFEA procedure was implemented into a two-dimensional, nonlinear finite element analysis program for RC, VecTor $2{ }^{8}$ This software, previously developed at the University of Toronto, is based on the DSFM and follows a rotating smeared-crack approach for modeling $\mathrm{RC}$. The program employs simple techniques for finite element modeling, using low order four-node rectangular, four-node quadratic, or three-node triangular elements for modeling $\mathrm{RC}$, while concentrating on the use of a wide array of advanced constitutive and behavioral models for concrete and steel reinforcement.

Taking advantage of the symmetric load and support conditions, only half of a test beam was modeled. A total of 992 rectangular elements were used to represent the concrete and 124 truss bar elements were used to model the longitudinal steel (Fig. 3). Mesh size was determined according to the past experience, by dividing the depth of the beam to 10 to 15 elements and keeping the aspect ratio smaller than 2.0. Some analyses were repeated with the number of elements approximately doubled to verify that the chosen mesh was not too coarse affecting the accuracy of the analyses. The transverse reinforcement was smeared within the concrete, because the stirrups were evenly distributed along the specimen. The steel plates provided at the supports and under the impact point were also included in the model. The specimens were restrained against upward movements at the supports; to simulate this condition, supports were introduced in the model with compression-only truss bars, so that the uplift forces were supported by the top truss bar, avoiding any artificial tensile stresses in the specimen that would occur if the bottom support was restrained against translations in the $y$-direction. These truss bars were assigned with the calculated stiffness properties of the supports.

The drop-weight was modeled with rectangular RC elements (Fig. 3). These elements were assigned a high rigidity, simulating the relatively nondeformable dropweight; they were connected to the structure with rigid compression-only truss bars, so that when the drop-weight bounced back, it would not pull up on the specimen. In other words, the drop-weight was disconnected from the structure after it bounced back during the analysis. The mass of the drop-weight was distributed equally to the nodes of the four elements representing the drop-weight, and the dynamic time step analysis was initiated with the contact velocity $(8.0 \mathrm{~m} / \mathrm{s})$ assigned to those nodes.

Except for the concrete compression base curve and the concrete hysteresis rules, VecTor2's default material and behavioral models ${ }^{8}$ were used in all analyses. The Popovics formulation ${ }^{15}$ for normal-strength concrete was selected as the compression base curve, because it was found to best fit the stress-strain curves obtained from the corresponding concrete test cylinders. Hysteresis response, which is critical

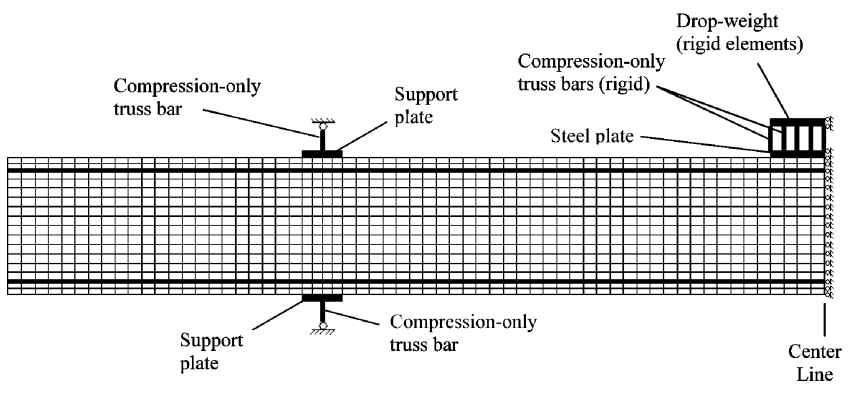

Fig. 3-Finite element model. in predicting the vibration properties and energy dissipation mechanisms, was simulated using the model proposed by Vecchio and Palermo. ${ }^{16}$ Using the reported mass data of the specimens, nodal masses were calculated and lumped to the nodes of the concrete elements. It has to be noted that the material models, including the concrete compressive and tensile behavior models, have not been modified to account for high strain rate effects. Although this is contrary to the findings obtained from numerous tests that concrete's observed strength increases with increasing strain rate, there is considerable recent discussion and disagreement among researchers concerning the inclusion of these strength gains in finite element modeling. Li and Meng, ${ }^{17}$ for example, argued that the strength gain observed in high strain rate tests was mostly due to the confining stresses resulting from the lateral inertia of the test specimens. Hence, there is a risk of overestimating the strength gain if finite element codes calculate the failure of concrete by using both strain rate dependent strength increase factors and confining stresses due the inertia. Later studies ${ }^{18,19}$ proved this point numerically, by modeling fictitious concrete specimens loaded under high strain rates and observing a strength increase with only considering the confining stresses due to the lateral inertia. The discussions on this subject are still premature, and more research is needed. In this study, however, the authors observed that including strength increase factors as recommended by $\mathrm{CEB}^{1}$ resulted in an unrealistically stiffer estimated response in the analyzed specimens. Because VecTor2 was capable of calculating the strength increase due to the confining stresses generated by the inertia of the structure, strain rate dependent strength increase factors were omitted in this study.

Although the structural damping was partly incorporated through the energy dissipation mechanisms inherent in the hysteresis models used, a minimal amount of viscous damping needed to be assigned to the specimens to ensure numerical stability. These damping ratios were decided by a parametric study, determining the minimum viscous damping required to obtain a numerically stable solution. Arbitrary damping ratios were assigned to the first and second modes to calculate the Rayleigh damping matrix, and the analyses were repeated with reduced damping ratios until the solutions lost stability. The smallest amount of viscous damping that resulted in a stable solution was selected for the final analyses of the test specimens. The damping ratios found sufficient for a stable analysis varied from 0.5 to $1 \%$ of critical damping assigned to the first two vibrational modes in accordance with the Rayleigh damping, with the exception of very heavily damaged specimens for which up to $5 \%$ damping ratio was needed.

In all analyses, Newmark's constant acceleration method $(\beta=0.25, \gamma=0.50)$ was used for its advantages in solution stability. The time step size selected for the numerical integration is critical in obtaining an accurate solution, especially for nonlinear structures. Hence, careful consideration should be given choosing the step size. One way to select a time step that would avoid unstable or inaccurate solutions is to perform an energy balance check. Any instability in the system creates spurious generation of energy, violating the conservation of energy. Hence, an energy balance check, as described by Belytschko et al., ${ }^{20}$ can detect this instability, even if it is local and not detectable by examining the results. If an instability is detected, the time step size should be reduced. An alternative and more practical way is to repeat the analysis with a time step half the original, and compare both solutions. If they are identical, the selected time step is acceptable; otherwise, it should be reduced. For the analyses 
presented in this study, the latter approach was followed to determine the time step size, yielding an optimal time step of 0.1 milliseconds. It has to be noted that, similar to the case regarding all other analysis parameters, the determination of neither the time step size nor the damping ratios require any comparison or calibration with respect to the measured response.

\section{COMPARISON OF FINITE ELEMENT SOLUTION AND EXPERIMENTAL RESULTS}

VecTor2 is a comprehensive finite element program, which gives extensive information about the structure analyzed, including concrete and reinforcement stresses, crack profiles, crack conditions, and reinforcement strains, in addition to common finite element analysis outputs such as displacements and support reactions. Here, for brevity, only midspan displacements, crack profiles, and some longitudinal reinforcement strains, as calculated by VecTor 2 and observed in the experiments, are compared.

Figure 4 presents the comparisons of the NLFEA calculations and the test results for the midspan displacements of the first impacts on undamaged specimens. Peak and residual values are summarized in Table 1. Note that the VecTor2 analysis of SSOb-1 is not included because, during the test, the specimen suffered failure-level damage beyond the analysis capabilities.

As seen in Fig. 4 and Table 1, NLFEA predictions for the midspan displacements of the test specimens were quite successful. Peak displacements were captured with considerable accuracy. Some discrepancies, however, were observed in the post-peak vibrations. For example, for most tests, the analyses predicted a somewhat shorter period of vibration compared to the test measurements. Damping for the specimens was underestimated in some lightly-damaged specimens (a-series) resulting in higher calculated displacement amplitudes, whereas it was overestimated for all heavily

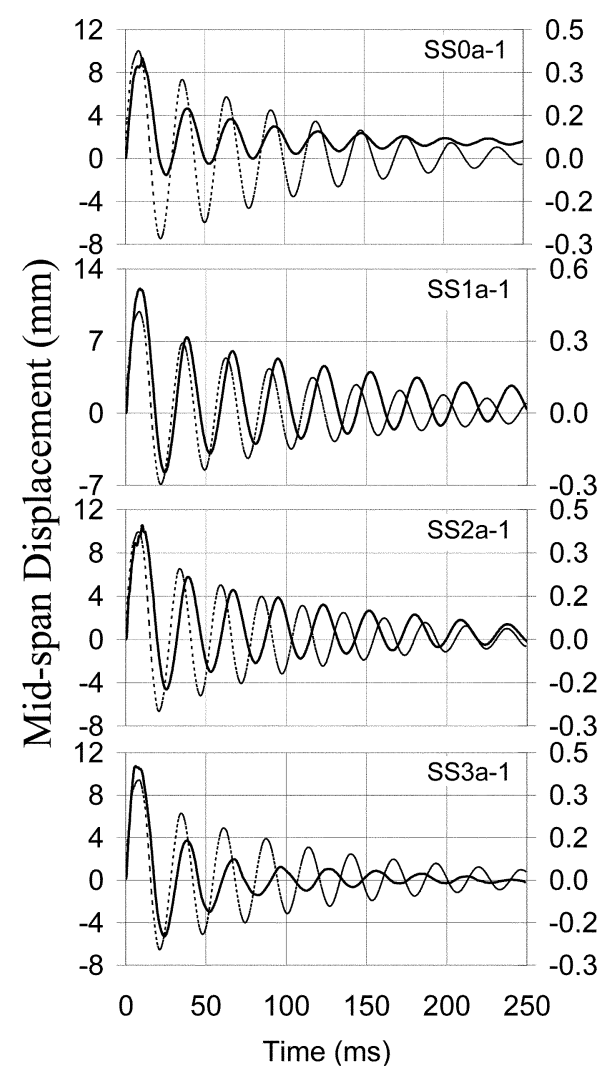

damaged specimens (b-series). On the other hand, peak displacements and post-peak vibrational periods were captured with greater accuracy for b-series specimens. In addition, residual displacements after the vibrations ceased were also captured accurately for all specimens.

For all analyses, crack profiles calculated by VecTor2 were visualized by a post-processor program called Augustus. ${ }^{21}$ Calculated and observed crack profiles for two tests, a heavily damaged specimen and a lightly damaged specimen, are compared in Fig. 5 and 6, respectively. It should be noted that, as a result of DSFM's rotating crack approach, the crack direction at a load stage is determined by the principal axis of stress calculated for that load stage. In other words, the direction of cracks constantly changes, and Augustus does not sketch the crack directions of the preceding load stages in the output. Therefore, a calculated crack profile does not reflect the cracking history of the structure, but, rather, relates only to that particular load stage.

Table 1-Comparison of displacements (first impacts)

\begin{tabular}{c|c|c|c|c|c|c}
\hline \multirow{2}{*}{ Test } & \multicolumn{3}{|c|}{ Peak displacements } & \multicolumn{2}{c}{ Residual displacements } \\
\cline { 2 - 7 } & $\begin{array}{c}\text { Test, } \\
\text { mm (in.) }\end{array}$ & $\begin{array}{c}\text { VecTor2, } \\
\text { mm (in.) }\end{array}$ & $\begin{array}{c}\text { Test/ } \\
\text { VecTor22 }\end{array}$ & $\begin{array}{c}\text { Test, } \\
\text { mm (in.) }\end{array}$ & $\begin{array}{c}\text { VecTor2, } \\
\text { mm (in.) }\end{array}$ & $\begin{array}{c}\text { Test/ } \\
\text { VecTor2 }\end{array}$ \\
\hline SS0a-1 & $9.3(0.36)$ & $10.0(0.39)$ & 0.93 & $1.6(0.06)$ & $0.3(0.01)$ & 5.33 \\
\hline SS1a-1 & $12.1(0.48)$ & $9.9(0.39)$ & 1.22 & $0.9(0.04)$ & $0.3(0.01)$ & 3.00 \\
\hline SS2a-1 & $10.0(0.39)$ & $9.9(0.39)$ & 1.01 & $0.5(0.02)$ & $0.2(0.01)$ & 2.50 \\
\hline SS3a-1 & $10.7(0.42)$ & $9.4(0.37)$ & 1.14 & 0.0 & 0.0 & 1.00 \\
\hline SS1b-1 & $39.5(1.56)$ & $34.6(1.36)$ & 1.14 & $17.7(0.70)$ & $17.5(0.69)$ & 1.01 \\
\hline SS2b-1 & $37.9(1.49)$ & $36.1(1.42)$ & 1.05 & $18.5(0.73)$ & $16.4(0.65)$ & 1.13 \\
\hline SS3b-1 & $35.3(1.39)$ & $34.9(1.37)$ & 1.01 & $17.7(0.70)$ & $17.8(0.70)$ & 0.99 \\
\hline \multicolumn{3}{c}{ Average } & 1.07 & - & 2.14 \\
\hline Coefficient of variance & 0.09 & - & 0.71 \\
\hline \multicolumn{3}{c}{}
\end{tabular}

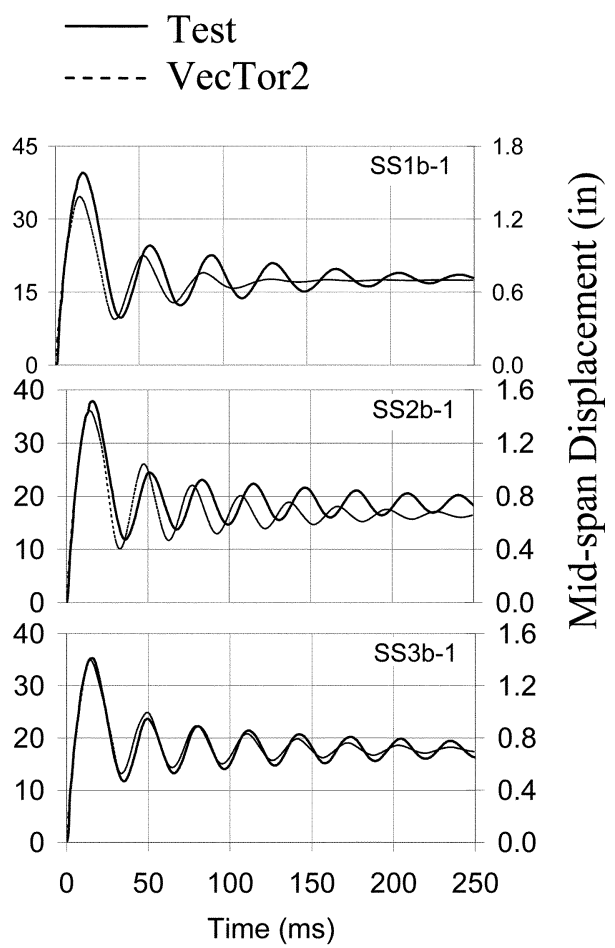

Fig. 4-Comparison of midspan displacements for first impacts on undamaged specimens. 
For this reason, several load stages need to be examined for a complete analysis of the estimated crack pattern. Moreover, the crack condition is calculated for each concrete element, whereas the cracks in the specimen would develop singly over a region. Hence, the calculated crack directions for individual elements should be regarded as an estimate of the inclination and width of a typical crack over that region. In Fig. 5 and 6, the crack profiles estimated at the initial stages of the response (negative moment phase as discussed by Saatci and $\operatorname{Vecchio}^{7}$ ), at the time the peak midspan displacement occurred, and at the final resting stage of the specimen, are presented and compared with the crack profiles obtained from the test results. Note that the crack widths measured after the tests relate to the final resting stage of the specimen. The crack widths calculated by VecTor2 for the time when the peak midspan displacement occurred are also presented to give an indication of the levels of predicted maximum crack widths.

As seen in the figures, the crack profiles were predicted well. Vertical cracks starting from the top face were predicted correctly at the early stages of the response. Vertical cracks in the beam overhangs, and their slight inclination towards the supports, were also predicted accurately. The NLFEA calculations support the idea that these cracks occur at the very early stages of the response, as argued by Saatci and Vecchio. ${ }^{7}$ In the later stages of the response, the formations of diagonal shear cracks were accurately predicted in both flexural-critical and shear-critical specimens.

\section{Table 2-Comparison of displacements} (second impacts)

\begin{tabular}{|c|c|c|c|c|c|c|}
\hline \multirow[b]{2}{*}{ Test } & \multicolumn{3}{|c|}{ Peak displacements } & \multicolumn{3}{|c|}{ Residual displacements } \\
\hline & $\begin{array}{c}\text { Test, } \\
\text { mm (in.) }\end{array}$ & $\begin{array}{l}\text { VecTor2, } \\
\text { mm (in.) }\end{array}$ & $\begin{array}{c}\text { Test/ } \\
\text { VecTor2 }\end{array}$ & $\begin{array}{l}\text { Test, } \\
\text { mm (in.) }\end{array}$ & $\begin{array}{l}\text { VecTor2, } \\
\text { mm (in.) }\end{array}$ & $\begin{array}{c}\text { Test/ } \\
\text { VecTor2 }\end{array}$ \\
\hline SS1a-2 & $39.6(1.56)$ & $45.3(1.78)$ & 0.87 & $17.5(0.69)$ & $17.0(0.67)$ & 1.03 \\
\hline SS2a-2 & $38.5(1.52)$ & $37.8(1.49)$ & 1.02 & $18.6(0.73)$ & $17.0(0.67)$ & 1.09 \\
\hline SS3a-2 & $36.3(1.43)$ & $34.4(1.35)$ & 1.06 & $17.0(0.67)$ & $15.4(0.61)$ & 1.10 \\
\hline SS2b-2 & $62.1(2.44)$ & $59.7(2.35)$ & 1.04 & $37.5(1.48)$ & $36.4(1.43)$ & 1.03 \\
\hline SS3b-2 & $54.8(2.16)$ & $53.8(2.12)$ & 1.02 & $33.3(1.31)$ & $30.8(1.21)$ & 1.08 \\
\hline \multicolumn{3}{|c|}{ Average } & 1.00 & \multicolumn{2}{|c|}{ - } & 1.07 \\
\hline \multicolumn{3}{|c|}{ Coefficient of variance } & 0.07 & \multicolumn{2}{|c|}{ - } & 0.03 \\
\hline
\end{tabular}

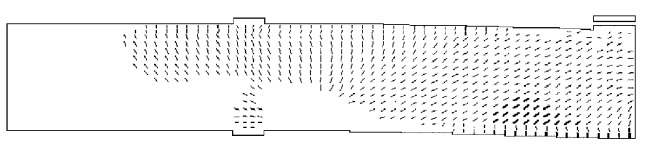

Calculated crack profile at $1 \mathrm{~ms}$

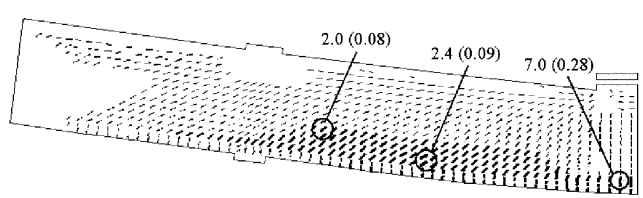

Calculated crack profile at $15 \mathrm{~ms}$ (peak displacement)

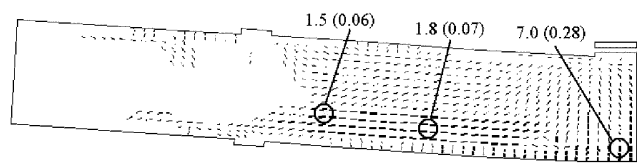

Calculated crack profile at the final resting stage

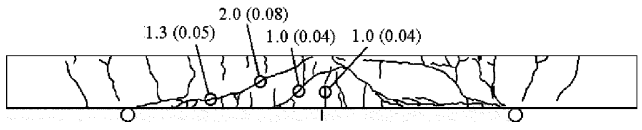

Observed crack profile

Fig. 5-Observed and calculated crack profiles, SS1b-1. (Note: Crack widths in mm [in.].)
Shear plug formations were also correctly represented in all heavily-damaged specimens.

Twelve strain gauges were connected to the longitudinal steel bars at different locations in the test specimens. Strains measured with these gauges were compared to the average reinforcement strain calculations obtained from NLFEA analyses. It has to be noted that strain gauge measurements on an embedded bar are highly sensitive to the proximity of the gauge to a crack. The smeared crack approach followed by the presented methodology allows calculating both an average strain and a local strain at a crack. Because the gauges close to the cracks mostly failed during testing, the measurements obtained from undamaged gauges were compared with calculated average strains. Herein, for brevity, only bottom longitudinal bar midspan strains were compared. As seen in Fig. 7, VecTor2 predicted the longitudinal reinforcement strains with reasonable accuracy. Peak strains were generally predicted with good accuracy, except for the highly damaged specimen in SS1b-1. Post-peak responses were also predicted well. The discrepancies in the comparisons can partly be attributed to the difficulties and errors involved in measuring the strains in a reinforcing bar, especially for the highly deformed specimens. Note that, in SS1b-1, the strains at the midspan were exceptionally high, possibly causing a loss in strain gauges' accuracy and resulting in the high discrepancy observed between the measured and computed responses.

Similar analyses were carried out for the second impact tests on the same specimens. VecTor2 has the capability to start from the damaged condition of a previous analysis and apply a new load on the structure. In such an analysis, initial conditions, such as displacements, concrete and reinforcement strains and stresses, and crack conditions are acquired from the final values of the previous analysis. The initial stiffness matrix $k_{0}$ for damping calculations is replaced with the stiffness matrix of the last time step of the previous analysis, and all proceeding stiffness calculations are carried out with consideration of the loading history of the structure. By using this feature, the second impacts on the same specimens were analyzed, starting from the results of the analyses carried out on the undamaged specimens. It should be noted that this approach causes accumulating errors, because the first analyses were not error-free, and, hence, these subsequent analyses start from a state that contains errors. On the other

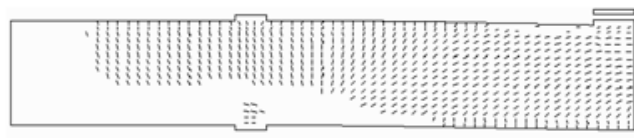

Calculated crack profile at $1 \mathrm{~ms}$

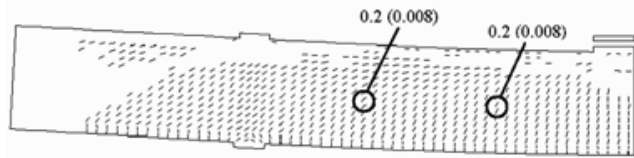

Calculated crack profile at $8 \mathrm{~ms}$ (peak displacement)

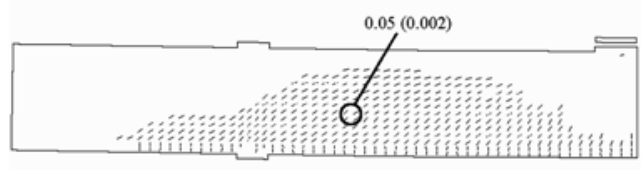

Calculated crack profile at the final resting stage

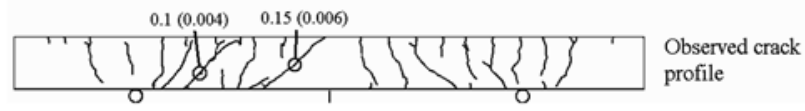

Fig. 6-Observed and calculated crack profiles, SS3a-1. (Note: Crack widths in mm [in.].) 


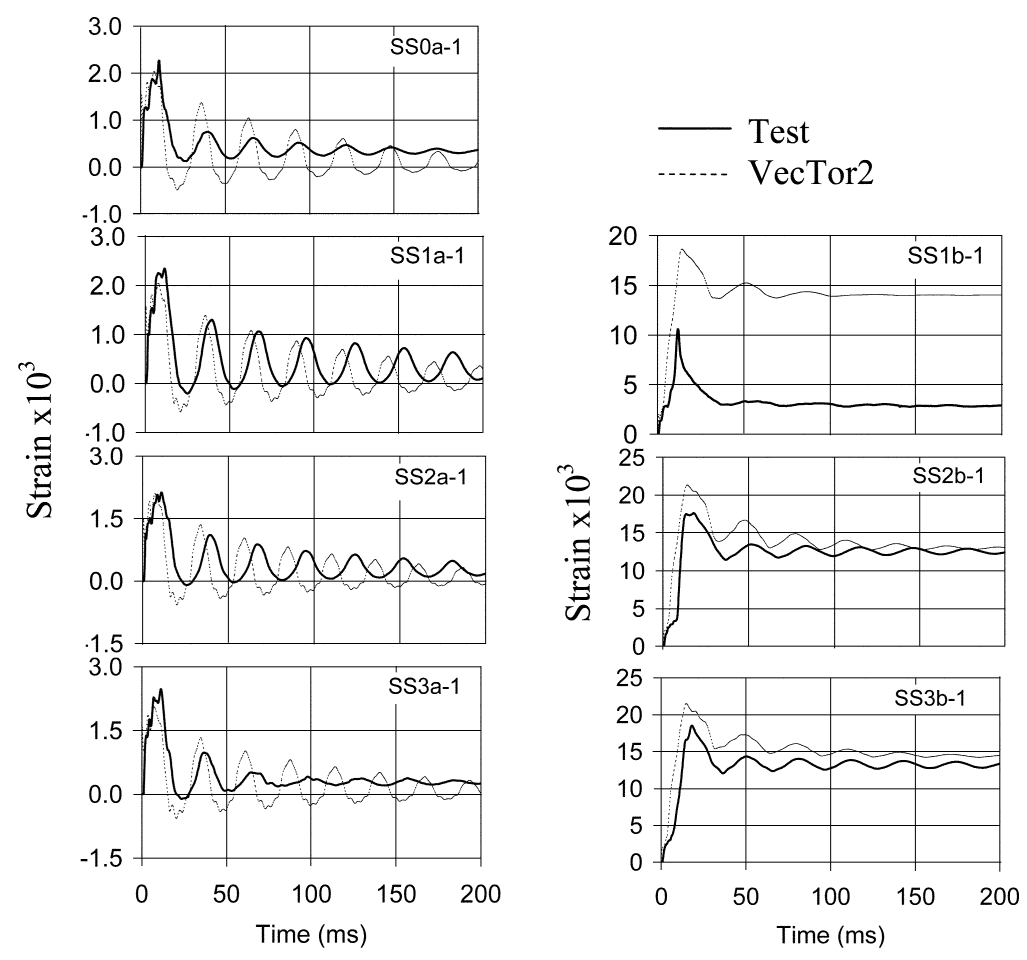

Fig. 7-Observed and calculated longitudinal reinforcement strains at midspan: first impacts.
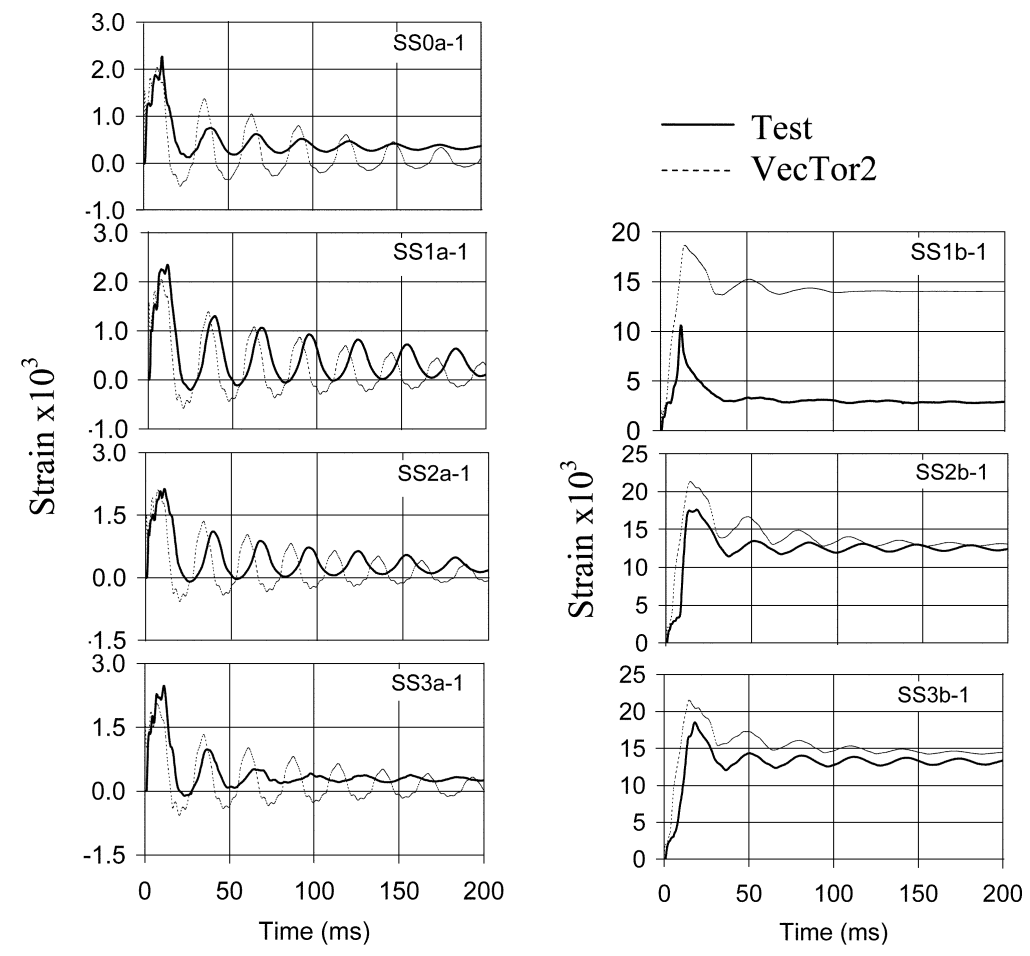

Fig. 8-Comparison of midspan displacements for second impacts on damaged specimens.

hand, the errors carried forward were likely not significant, evidenced by the good accuracy in residual displacements obtained from the first analyses, allowing for stable solutions with reasonable accuracies. Attempts to analyze the same specimens for the third impact tests, however, generally failed because the accumulated errors from the first and second impact analyses, coupled with the numerical problems related to the significantly reduced stiffnesses of the highly damaged specimens after the first two impact tests, made it impossible to obtain a numerically stable solution. Second impact Tests SSOa-2 and SS1b-2 were also omitted, because in these tests, the specimens suffered extensive damage beyond the analysis capabilities of VecTor2.

Figure 8 compares the midspan displacements as measured during the tests and found from VecTor 2 analyses. As summarized in Table 2, the agreement between the test 
results and the VecTor2 calculations were very satisfactory, with discrepancies less than $10 \%$ for most tests, both for peak and residual displacements. In general, however, the calculated response dampened out faster than the measured response. In other words, as seen in some analyses carried out for the first impacts, VecTor2 overestimated the damping in the specimens.

The crack profiles calculated by VecTor 2 are compared in Fig. 9 and 10 with the profiles obtained from the second tests. Only the results for two tests, SS3a-2 representing a lightly damaged specimen, and $\mathrm{SS} 2 \mathrm{~b}-2$ representing a heavily damaged specimen, are presented here for brevity. As seen in the figures, the NLFEA analyses predicted general crack patterns reasonably well. Extensive cracking at the level of bottom reinforcement was predicted accurately; note that most specimens either developed horizontal cracks at this level, or they suffered spalling of the bottom cover concrete. Wide diagonal cracks forming the shear-plug were also predicted successfully, including the statically flexuralcritical specimens. In the tests, it was observed that the

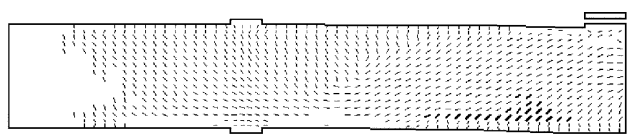

Calculated crack profile at $1 \mathrm{~ms}$

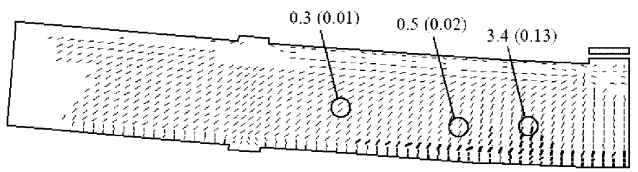

Calculated crack profile at $15 \mathrm{~ms}$ (peak displacement)

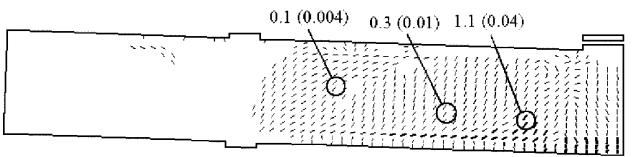

Calculated crack profile at the final resting stage

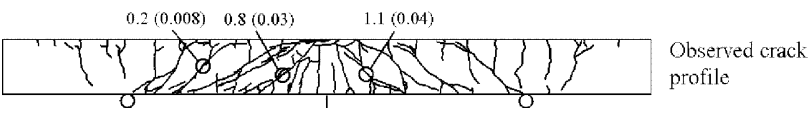

Fig. 9-Observed and calculated crack profiles, SS3a-2. (Note: Crack widths in mm [in.].)

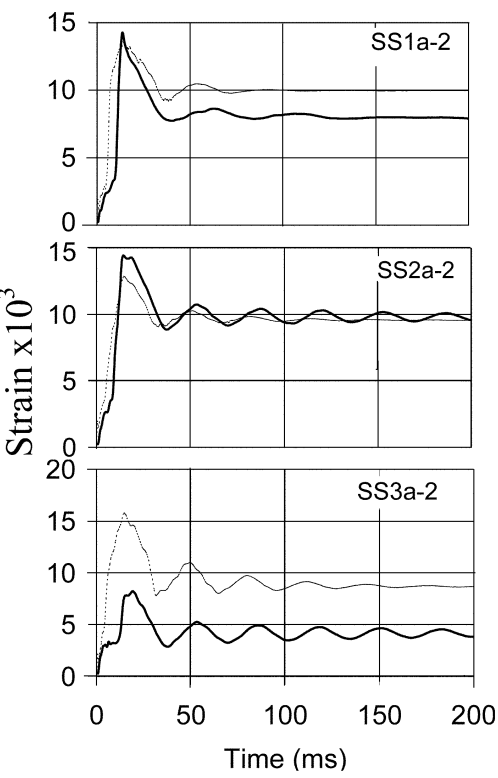

diagonal cracks forming the shear-plug were wider close to the bottom; this also was observed in the calculations. Some discrepancies, however, exist in the crack width predictions. For example, VecTor2 over-estimated the residual widths of the vertical cracks at the midspan.

The comparison of the test and analysis results for the midspan longitudinal reinforcing bar strains are presented in Fig. 11 for the second impacts. As seen in the figure, VecTor2 predicted longitudinal reinforcement strains at the midspan with reasonable accuracy. Although VecTor2 generally overestimated the strain response, the general shapes of calculated and measured strain responses were sufficiently similar. In general, it seems that the factors that caused the discrepancies between the calculated and measured response in the first impact test analyses persisted for the second impact analyses as well. Nevertheless, considering the high levels of damage the specimens suffered, it is significant that a NLFEA analysis estimated the displacements, crack profiles, and reinforcing bar strains with very good levels of accuracy.

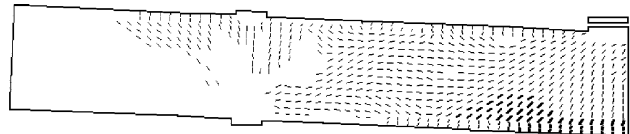

Calculated crack profile at $1 \mathrm{~ms}$

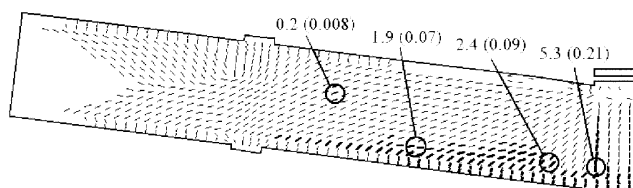

Calculated crack profile at $15 \mathrm{~m}$ (peak displacement)

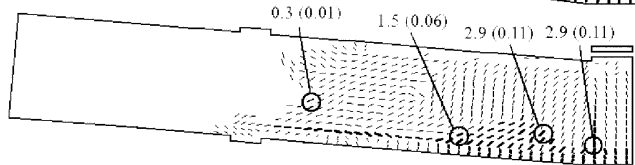

Calculated crack profile at the final resting stage

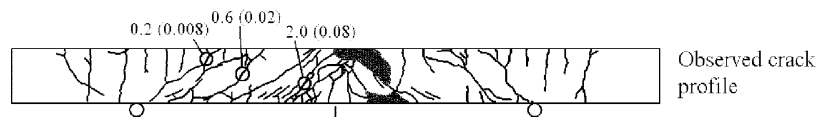

Fig. 10-Observed and calculated crack profiles, SS2b-2. (Note: Crack widths in $\mathrm{mm}$ [in.].)

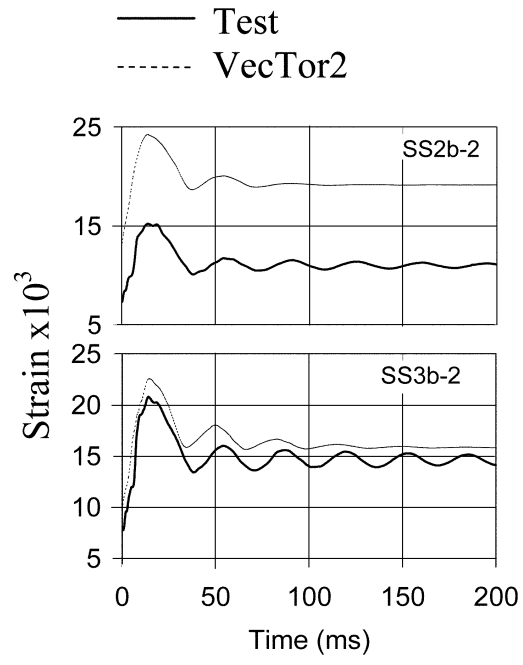

Fig. 11-Observed and calculated longitudinal reinforcement strains at midpan, second impacts. 


\section{CONCLUSIONS}

Comparisons of beam impact test results with VecTor2 analysis results using the proposed NLFEA procedure led to the following conclusions:

1. The proposed NLFEA procedure performed well in predicting displacements, damage levels, and reinforcement strains. The methodology based on the DSFM proved to be successful in predicting the shear-dominant behavior of the specimens under impact loads. The shear-critical failure under dynamic loading of statically flexural-critical specimens was captured well;

2. Post-peak vibration characteristics, compared between the test results and the NLFEA analyses' results, showed some minor discrepancies. These discrepancies were mostly attributed to the deficiencies regarding the modeling of hysteresis behavior of concrete and steel under high strain rate conditions;

3. The proposed NLFEA procedure was also successful in predicting the response of the damaged specimens for the second impact tests on the same specimens. Agreement between the test and analysis results for the peak and residual displacements was strong. This indicates that the proposed procedure and the DSFM are capable of accurately modeling the reduced stiffness of a reinforced concrete structure after it suffers considerable levels of damage;

4. The simplified approach followed for modeling the drop-weight and impact load proved to be successful. This approach completely eliminated the need for an impact force history. Predetermination of the impact force history in an impact event generally requires employing sophisticated methods; however, the only required parameters for the impact analysis with the proposed procedure were the dropweight mass and its impact velocity, which are usually known parameters for design problems;

5 . The proposed procedure, in conjunction with the chosen behavioral models, incorporated the majority of the energy dissipating mechanisms in to the structural model. Only a slight amount of viscous damping, added through the Rayleigh method, was needed to numerically stabilize the solutions. The majority of the damping was supplied by other behavioral mechanisms such as material hysteresis and concrete cracking. The fact that externally assigned viscous damping had little role in the calculated response is important, because it is difficult to estimate the damping characteristics of a structure. Modeling of energy dissipating mechanisms by VecTor2 significantly reduced the role of an uncertain parameter in the computer model. On the other hand, hysteresis models for concrete and steel should be improved for high strain rate loadings to better simulate the entire damping characteristics of the structure;

6. The proposed procedure was computationally efficient. The time required to complete an analysis was typically significantly shorter than with some other well-known sophisticated finite element programs. Using short time steps for improved accuracy did not have a severe adverse effect on computational times because iterations converged faster with shorter time steps;

7. Omitting the strain rate dependent strength increase factors for concrete resulted in more accurate predictions for the analyzed test specimens. This issue, however, needs to be further investigated with different tests, strain rates, and strain rate dependent material formulations, to determine if it was a situation particular to the test specimens analyzed, or the CEB formulations used for considering the strain rate effects were overestimating the strength gain; and
8. The proposed methodology is currently not capable of modeling the local damage, such as penetration, perforation, or scabbing under high-velocity impacts. Attempts to simulate the tests on heavily damaged specimens, such as SSOb-1, SSOa-2, and SS1b-2 that developed cracks wider than $20 \mathrm{~mm}$ (0.8 in.) and suffered extensive scabbing, have also failed due to the numerical instabilities and the limitations of the behavioral models employed. Further improvements are needed in both the methodology and the behavioral models to extend the analysis capabilities to include the failure-level damages.

\section{REFERENCES}

1. Comité Euro-International du Béton, "Concrete Structures under Impact and Impulsive Loading," Bulletin D'Information, No. 187, 1988.

2. Krauthammer, T.; Shahriar, S.; and Shanaa, H. M., "Response of Reinforced Concrete Elements to Severe Impulsive Loads," Journal of Structural Engineering, V. 116, No. 4, 1990, pp. 1061-1079.

3. Bischoff, P. H.; Perry, S. H.; and Eibl, J., "Contact Force Calculations with a Simple Spring-Mass Model for Hard Impact: A Case Study Using Polystyrene Aggregate Concrete," International Journal of Impact Engineering, V. 9, No. 3, 1990, pp. 317-325.

4. Hamouda, A. M. S., and Hashmi, M. S. J., "Modeling the Impact and Penetration Events of Modern Engineering Materials: Characteristics of Computer Codes and Material Models," Journal of Materials Processing Technology, V. 56, No. 1, 1996, pp. 847-862.

5. Kishi, N.; Mikami, H.; and Ando, T., "An Applicability of Impact FE Analysis on Shear-Failure-Type RC Beams with Shear Rebar," Proceedings of the 4th Asia-Pacific Conference on Shock and Impact Loads on Structures, Singapore, 2001, pp. 309-315.

6. Kishi, N.; Mikami, H.; Matsuoka, K.G.; and Ando, T., "Impact Behavior of Shear-Failure-Type RC Beams without Shear Rebar," International Journal of Impact Engineering, V. 27, No. 9, 2002, pp. 955-968.

7. Saatci, S., and Vecchio, F. J., "Effects of Shear Mechanisms on Impact Behavior of Reinforced Concrete Beams," ACI Structural Journal, V. 106, No. 1, Jan.-Feb. 2009, pp. 78-86.

8. Vecchio, F. J., and Wong, P., VecTor2 and Formworks Manual, Publication No. 2002-02, Department of Civil Engineering Publication, University of Toronto, Toronto, ON, Canada, 2002.

9. Vecchio, F. J., "Disturbed Stress Field Model for Reinforced Concrete: Formulation," Journal of Structural Engineering, V. 126, No. 9, 2000, pp. 1070-1077.

10. Vecchio, F. J., and Collins, M. P., "The Modified CompressionField Theory for Reinforced Concrete Elements Subjected to Shear," ACI Journal, Proceedings V. 83, No. 2, Mar.-Apr. 1986, pp. 219-231.

11. Chopra, A. K., Dynamics of Structures, Prentice Hall, Inc., Upper Saddle River, NJ, 2001.

12. Garbow, B. S., "EISPACK-A Package of Matrix Eigensystem Routines," Computer Physics Communications, V. 7, 1974, pp.179-184.

13. Newmark, N. M., "A Method of Computation for Structural Dynamics," Journal of the Engineering Mechanics Division, ASCE, V. 85, 1959, pp. 67-94.

14. Saatci, S., "Behaviour and Modeling of Reinforced Concrete Structures Subjected to Impact Loading," PhD thesis, Department of Civil Engineering, University of Toronto, Toronto, ON, Canada, 2007.

15. Popovics, S., "A Numerical Approach to the Complete Stress-Strain Curve of Concrete," Cement and Concrete Research, V. 3, No. 5, 1973, pp. 583-599.

16. Palermo, D., and Vecchio, F. J., "Compression Field Modeling of Reinforced Concrete Subjected to Reversed Loading: Formulation," ACI Structural Journal, V. 100, No. 5, Sept.-Oct. 2003, pp. 616-625.

17. Li, Q. M., and Meng, H., "About the Dynamic Strength Enhancement of Concrete-Like Materials in a Split Hopkinson Pressure Bar Test," International Journal of Solids and Structures, V. 40, 2003, pp. 343-360.

18. Cotsovos, D. M., and Pavlovic, M. N., "Numerical Investigation of Concrete Subjected to Compressive Impact Loading-Part 1: A Fundamental Explanation for the Apparent Strength Gain at High Loading Rates," Computers and Structures, V. 86, 2008, pp. 145-163.

19. Cotsovos, D. M., and Pavlovic, M. N., "Numerical Investigation of Concrete Subjected to High Rates of Uniaxial Tensile Loading," International Journal of Impact Engineering, V. 35, 2008, pp. 319-335.

20. Belytschko, T.; Liu, W. K.; and Moran, B., Nonlinear Finite Elements for Continua and Structures, John Wiley \& Sons, England, 2000.

21. Bentz, E. C., Augustus: Finite Element Post-Processor for VecTor2 and TRIX, University of Toronto, Toronto, ON, Canada, 2003. 
Reproduced with permission of the copyright owner. Further reproduction prohibited without permission. 